\title{
Review of the antiviral activity and pharmacology of monoglycerides and implications for treatment of COVID-19
}

\author{
Mira Aldridge ${ }^{1}$ \\ ${ }^{1}$ correspondence: miraaldridge@gmail.com
}

\begin{abstract}
:
Monoglycerides are a class of lipids derived from fatty acids with well established antiviral properties. Monoglycerides are widely used in food processing, cosmetics and in nutritional supplements. This review examines the biochemical and pharmacological properties of monoglycerides and fatty acids as they pertain to COVID-19 pathogenesis. Information on monoglycerides, fatty acids and COVID-19 is presented with sources gathered through PubMed, Elsevier, JSTOR, Oxford University Press Medicine Archive, Wiley, MedRxiv and Google. The antiviral activity of monoglycerides has been documented since the 1970's. In vitro studies have shown that viral concentrations of enveloped viruses were reduced by over 10,000 fold in the presence of monoglycerides such as glycerol monolaurate. Moreover, glycerol monolaurate is widely available and generally recognized as safe (GRAS) by the FDA. The aim of this review is to present an evidence based case for further investigation into this class of compounds for the potential treatment of COVID-19.
\end{abstract}

Keywords: glycerol monolaurate, monolaurin, fatty acids, monoglycerides, lipids, enveloped virus, antiviral, antibacterial, antimicrobial, pathogenesis, COVID-19, ARDS, T-Cells, immune, cytokine, amphiphilic, IL-6

\section{Introduction}

The COVID-19 pandemic is a fast evolving public health and economic emergency. Currently there is no approved vaccine and no preventative medical interventions in use to slow the spread of the disease. Unfortunately long vaccine development timelines preclude their usefulness in alleviating the initial consequences of pandemics. Existing pharmaceutical antiviral or reappropriated drugs may prove effective in reducing severity and length of infection, but substantiative evidence is lacking at this time. If a successful pharmaceutical intervention is found it is unlikely that supply and safety profiles will allow for widespread use as a prevention 
method. As asymptomatic cases and long delays in the onset of symptoms are two of the forces driving rapid transmission, addressing these populations may have a significant impact on the trajectory of the pandemic. Therefore pursuing development or discovery of broad spectrum antivirals would be advantageous at this time. In view of this, several naturally occurring biological compounds have demonstrated antiviral activity in vitro and in vivo, which may prove to be beneficial in the treatment and prevention of COVID-19. This paper will review the pharmacological and antiviral data of monoglycerides, namely glycerol monolaurate, as it is relevant to COVID-19 pathogenesis and treatment. Lastly, the investigations of this review may prove useful for future novel virus outbreaks or for other medical applications.

\section{Background}

Monoglycerides are an important group of surfactants produced by the interesterification of fats or oils with glycerol. Monoglycerides are widely used in the food industry and are derived from several common fatty acids such as lauric, linoleic, myristic, oleic, palmitic, and stearic acids. Monoglycerides are prepared from food sources of edible oils such as coconut oil. According to the FDA, functions of monoglycerides as a food additive or ingredient include: emulsifier, lubricant and release agent, solvent, stabilizer and thickener and a surface reactive agent. Monoglycerides are generally recognized as safe (GRAS) by the FDA and are permitted for use in food with no limitation [1]. Glycerol monolaurate, the monoglyceride derivative of the medium chain saturated fatty acid lauric acid, is also available as a nutritional supplement.

Fatty acids were discovered by the french chemist Chevreul in the 1820's. In studying the saponification process, Chevreul discovered, contrary to previous belief, that soap was composed of an alcohol, which he called glycerin, and a fatty acid salt. This pioneering research introduced the term fatty acid to the scientific community and led to the acceptance of this important new class of compounds in organic chemistry [2]. Antimicrobial activity was first noted in fatty acids in the late 1800's when Koch observed that fatty acids inhibited the growth of Bacillus anthracis [3]. In the 1940's Burtenshaw discovered endogenous antimicrobial lipids in human skin that played a role in the skin's immune system [4]. After the advent of antibiotics, antimicrobial lipids were largely ignored until the 1970's when Kabara methodically investigated the antibacterial properties and potencies of various fatty acids and their derivatives [5]. Since then, extensive research has been done on the antimicrobial properties of fatty acids and monoglycerides. Fatty acids are classified by their chain length and degree of saturation. Certain fatty acids and their monoglyceride derivatives have demonstrated varying degrees of antimicrobial activity against bacteria, enveloped viruses and fungi [3, 5 - 26]. 


\section{3a. Cytokine storm in COVID-19}

We now understand the main cause of death in COVID-19 patients is likely attributed to Acute Respiratory Distress Syndrome (ARDS). ARDS is a condition whereby fluid builds up in air sacs in the lungs preventing oxygenation of the blood and eventually leading to respiratory failure. COVID-19 patients arrive at this point via the pathway known as the cytokine storm, which causes a flood of pro inflammatory cytokines directed by the immune system at bodily tissues causing extreme inflammation, organ failure and death. Elevated levels of many cytokines such as IFN- $\alpha$, IFN- $\gamma$, IL-1 $\beta$, IL-6, IL-12, IL-18, IL-33, TNF- $\alpha$ and TGF $\beta$ are released in the cytokine storm [27]. Recent analysis by Herold and colleagues showed that the inflammatory marker IL-6 is a highly accurate predictive correlator of the need for mechanical respiration and respiratory failure in hospitalized COVID-19 patients [28]. Other studies have confirmed the correlation of elevated IL-6 with severity of disease [29, 30] and, in addition, noted that lowered IL-6 levels were closely related to treatment effectiveness and recovery [30].

\section{3b. Covid-19 immune evasion}

$\mathrm{Li}$ et al. have summarized several of the immune evasion strategies in coronaviruses such as SARS-CoV and MERS-CoV in an effort to predict the immune evasion techniques of the novel COVID-19 virus. SARS-CoV and MERS-CoV are able to replicate in special double membrane vesicles that disguise their standard pathogen-associated molecular patterns (PAMPS) thereby evading detection by the innate immune system. These coronaviruses inhibit INF-I which would otherwise have a protective effect against the viruses, and they have also been found to downregulate gene expression related to antigen presentation, theoretically interfering with the adaptive immune response [27].

\section{Biochemical features of fatty acids and monoglycerides}

Monoglycerides are a class of lipids derived from fatty acids. Monoglycerides are composed of a glycerol molecule linked to a fatty acid via an ester bond. The glycerol head of the molecule is hydrophilic while the fatty acid tail of the molecule is hydrophobic, making it an amphiphilic molecule.

\section{4a. Antiviral activity}

The antiviral activity of various naturally occuring fatty acids and their monoglycerides has been demonstrated [6 - 13], most notably in studies assessing the antimicrobial properties of human milk [6, 8 - 10, 31]. In vitro studies by Thormar, Isaacs et al. in the 1980's and 1990's showed strong antiviral effects of free fatty acids and their monoglycerides on the enveloped viruses 
vesicular stomatitis virus, herpes simplex virus, visna virus, and HIV [6, 7]. Some fatty acids, primarily medium chain saturated fatty acids and long chain unsaturated fatty acids, were able to reduce viral concentrations of VSV and HSV in cell cultures by 10,000 fold. Among medium chain saturated fatty acids, lauric acid exhibited this magnitude of antiviral activity at the lowest concentrations. Moreover the researchers determined that the monoglycerides of these fatty acids had greater powers of viral inactivation than the free fatty acids. Where they were effective, monoglycerides had equal capacity for viral inactivation at 5 to 10 times lower concentrations than their corresponding fatty acids. Glycerol monolaurate, the monoglyceride of lauric acid, was the one of most active against the enveloped viruses in these studies. No fatty acids or monoglycerides were able to inactivate polio virus, a nonenveloped virus [6].

Contributing to a fuller understanding of the antiviral action of fatty acids and monoglycerides, Hillmarsson et al. showed that viruses differ in sensitivity to lipids. In observing the effects of various fatty acids, fatty alcohols, and monoglycerides on HSV1 and HSV2, the researchers noted that HSV was more susceptible to monocaprin but was still inactivated by monolaurin (glycerol monolaurate) over a longer time period. Both viruses were even more sensitive to lauric acid, capric acid and fatty alcohols however the cytotoxicity of some of these substances exclude usefulness in humans [11]. It has been noted, however, that topical monoglycerides, such as monocaprin, have been well tolerated in studies on mice [11, 32].

Studies on the antimicrobial effects of human milk have also demonstrated the antiviral activity of monoglycerides on enveloped viruses. Isaacs, Thormar et al. showed that milk aspirated from infant stomachs 1 to 3 hours after feeding were able to reduce viral counts of herpes simplex virus and vesicular stomach virus by as much as 10,000 fold. The researchers attributed these results to the intragastric formation of fatty acids and their monoglycerides from the triglyceride components of milk [8 - 10].

\section{4b. Membrane lytic behavior}

The antiviral effect of monoglycerides and fatty acids has been attributed to their disruptive effect on lipid membranes. Isaacs, Thormar, et al. observed that fatty acid and monoglyceride solutions created leaks in the viral membrane and, in higher concentrations, resulted in complete destruction of viral membranes and cell lysis. They suggested that fatty acids and monoglycerides may be incorporated into the viral lipid membrane, creating a destabilizing effect in the lipid bilayer [6].

The membrane lytic behavior of fatty acids and monoglycerides has also been widely documented in studies investigating these substances for their antibacterial properties [14 - 24]. These studies yield additional insight into the membrane disrupting mechanisms of fatty acids 
and monoglycerides. In bacterial membranes, several effects have been observed in response to fatty acids or monoglycerides, including membrane destabilization, pore formation, increased permeability, disruption of electron transport chain, interruption of oxidative phosphorylation, and the inhibition of bacterial membrane associated enzymes [3].

Several researchers have proposed that lipids are integrated into the lipid membranes of pathogenic organisms thereby disrupting functioning [3, 6, 15, 33]. It has been suggested this integration may happen as a result of the amphipathic and lipophilic properties of fatty acids and monoglycerides $[3,15]$.

\section{Pharmacological effects of glycerol monolaurate}

In vitro and in vivo studies have shown various immune modulating and anti-inflammatory effects in cells in response to glycerol monolaurate (GML). At lower concentrations, GML appeared to boost immune response via increased $\mathrm{T}$ cell lymphocyte production. However at higher concentrations, T cell lymphocyte proliferation was inhibited. The immunosuppressive effects of GML had no effect on B cell lymphocyte proliferation [34]. Several pro-inflammatory cytokines were also inhibited by GML in vitro and in vivo. Various studies have observed reduced production of cytokines and chemokines such as IL- 2, IFN- $\gamma$, TNF- $\alpha$, IL- $1 \alpha$, IL- $1 \beta$ MIP-3 $\alpha$, IL-6, and IL-8 in response to GML [25, 26, 34 - 36]. Zhang et al. have mechanistically attributed this effect to defective signaling due to altered lipid dynamics in $\mathrm{T}$ cell membranes. They have proposed, as a result of suppressed $\mathrm{T}$ cell activation and reduced cytokine production, that GML may serve as a potent immunosuppressive agent [35]. In one in vivo study investigating Simian Immunodeficiency Virus transmission in rhesus macaques, researchers not only prevented SIV transmission in a small sample with topical application of GML, they also showed reduced levels of two proinflammatory markers, MIP-3 $\alpha$ and IL-8, from cervical samples collected [36].

\section{Discussion}

It has been proposed that there may be several immune evasion techniques utilized by COVID-19 to avoid immune responses. These mechanisms point to a complex picture regarding the ability of the immune system to fully eradicate the virus. Such factors may contribute to the large number of asymptomatic cases and the delay in onset of symptoms, and underlie the necessity of addressing these populations to combat further spread of the pandemic.

The antiviral properties of monoglycerides have been linked to their membrane disrupting properties. Some researchers have suggested the molecules may be integrated into viral and other phospholipid membranes [3, 6, 15,33] causing destabilization [6], and others have further 
illustrated this property, explaining that this capacity stems from its amphiphilic nature $[3,15]$. Interestingly soap molecules are also amphilitic in nature. This distinctive feature of the soap molecule is the biochemical characteristic that gives soap its powerful bacteria and virus destroying capacities. It is also the same characteristic that allows monoglycerides to act as emulsifying agents in food preparation. Furthermore, in reviewing the literature and data it is difficult to ignore the origin of the discovery of fatty acids: from the soap making process. This connection may prove an important clue in furthering our understanding of monoglycerides and their broad spectrum antimicrobial effects.

There have currently been no studies on the effectiveness of any monoglycerides in COVID-19 patients; however, COVID-19 is an enveloped virus with a protective lipid envelope. Given the substantial evidence of antiviral action of monoglycerides on enveloped viruses, it follows, therefore, that glycerol monolaurate, or another monoglyceride, is likely to have a destructive effect on COVID-19 protective membranes. In fact, recent proposals regarding COVID-19 have suggested that disruption of the lipid structures and consequent impairment of viral attachment to host cells is a possible strategy of reducing infectivity in COVID-19 patients [37].

Recent data released indicates that elevated levels of the inflammatory marker IL-6 is highly correlated with illness severity in COVID-19 patients [28-30]. In addition to its antiviral effects, glycerol monolaurate has been shown to reduce inflammatory immune responses under certain conditions, including suppressing IL-6 [26]. On account of these effects, glycerol monolaurate may have an inhibitory effect on the overactive immune response observed during cytokine storm conditions in severe COVID-19 cases. More research is needed to determine the magnitude of these immune effects in vivo and how outcomes are affected in respect to the cytokine storm.

\section{Conclusion}

Glycerol monolaurate is widely available as a nutritional supplement and generally recognized as safe by the FDA. If found effective, monoglycerides such as glycerol monolaurate may have a beneficial effect on the trajectory of the COVID-19 pandemic.

\section{Limitations}

There are several limitations to this review, primarily the lack of clinical studies in humans. However, in light of the extensive safety profile and potential wide ranging implications, the scientific community would benefit from further research into the efficacy of monoglycerides in the treatment or prevention of COVID-19. 
Competing interest statement: The author declares no competing interest.

Funding: There was no funding for this review.

\section{References:}

1. 21.CFR.184.1505. Title 21. Food and drugs. Chapter I. Food and Drug Administration. Subchapter B. Food for human consumption. Part 184. Direct food substances affirmed as generally recognized as safe. Subpart B. Listing of specific substances affirmed as GRAS. 21 CFR 184.1505 - mono- and diglycerides. https://www.ecfr.gov/cgi-bin/text-idx?SID=0892a3ff41f17f9660183ec0cdf21f94\&mc=tr $\underline{\text { ue } \& \text { node }=\text { se21.3.184 11505\&rgn }=\operatorname{div} 8}$ (accessed: April 6, 2020)

2. Costa, Albert. Encyclopaedia Britannica, s.v. "Michel-Eugène Chevreul." Chicago: Encyclopaedia Britannica, 2020. https://www.britannica.com/biography/Michel-Eugene-Chevreul (accessed: April 6, 2020)

3. Yoon BK, Jackman JA, Valle-González ER, Cho N-J, Yoon BK. Antibacterial Free Fatty Acids and Monoglycerides: Biological Activities, Experimental Testing, and Therapeutic Applications. International journal of molecular sciences. 2018;19(4). doi:10.3390/ijms19041114

4. Burtenshaw JML, BURTENSHAW JML. Self-disinfection of the skin; a short review and some original observations. British medical bulletin. 1945;3(7-8):161-164. doi:10.1093/oxfordjournals.bmb.a071901

5. Kabara JJ, Swieczkowski DM, Conley AJ, Truant JP. Fatty Acids and Derivatives as Antimicrobial Agents. Antimicrobial Agents and Chemotherapy. 1972;2(1):23-238. doi:10.1128/AAC.2.1.23

6. Thormar H, Isaacs CE, Brown HR, Barshatzky MR, Pessolano T. Inactivation of enveloped viruses and killing of cells by fatty acids and monoglycerides. Antimicrobial Agents and Chemotherapy. 1987;31(1):27-31. doi:10.1128/AAC.31.1.27

7. Isaacs $\mathrm{CE}$, Kim KS, Thormar, H. Inactivation of enveloped viruses in human bodily fluids by purified lipids. Annals of the New York Academy of Sciences. 1994 June6; 724:457-64. doi:10.1111/j.1749-6632.1994.tb38947.x

8. Isaacs CE, Litov RE, Thormar H. Antimicrobial activity of lipids added to human milk, infant formula, and bovine milk. The Journal of Nutritional Biochemistry. 1995;6(7):362-366. doi:10.1016/0955-2863(95)80003-U 
9. Isaacs CE, Kashyap S, Heird WC, Thormar H. Antiviral and antibacterial lipids in human milk and infant formula feeds. Archives of Disease in Childhood. 1990;65(8):861-864. doi:10.1136/adc.65.8.861

10. Isaacs CE, Thormar H, Pessolano T. Membrane-disruptive effect of human milk: inactivation of enveloped viruses. The Journal of infectious diseases. 1986;154(6):966-971. doi:10.1093/infdis/154.6.966

11. Hilmarsson H, Kristmundsdóttir T, Thormar H. Virucidal activities of medium- and long-chain fatty alcohols, fatty acids and monoglycerides against herpes simplex virus types 1 and 2: comparison at different pH levels. APMIS. 2005;113(1):58-65. doi:10.1111/j.1600-0463.2005.apm1130109.x

12. Hilmarsson H, Larusson LV, Thormar H. Virucidal effect of lipids on visna virus, a lentivirus related to HIV. Archives of Virology. 2006;151(6):1217-1224.

doi:10.1007/s00705-005-0699-2

13. Sands JA. Inactivation and Inhibition of Replication of the Enveloped Bacteriophage \{varphi\} 6 by Fatty Acids. Antimicrobial Agents and Chemotherapy. 1977;12(4):523-528. doi:10.1128/AAC.12.4.523

14. Speert DP, Wannamaker LW, Gray ED, Clawson CC. Bactericidal Effect of Oleic Acid on Group A Streptococci: Mechanism of Action. Infection and Immunity. 1979;26(3):1202-12010. doi:10.1128/IAI.26.3.1202-1210.1979

15. Hyldgaard M, Sutherland DS, Sundh M, Mygind T, Meyer RL, Hyldgaard M. Antimicrobial mechanism of monocaprylate. Applied and environmental microbiology. 2012;78(8):2957-2965. doi:10.1128/AEM.07224-11

16. Knapp HR, Melly MA, Knapp HR. Bactericidal effects of polyunsaturated fatty acids. The Journal of infectious diseases. 1986;154(1):84-94. doi:10.1093/infdis/154.1.84

17. Wang LL, Johnson EA. Inhibition of Listeria monocytogenes by fatty acids and monoglycerides. Applied and Environmental Microbiology. 1992;58(2):624-629. doi:10.1128/AEM.58.2.624-629.1992

18. Bergsson G, Arnfinnsson J, Karlsson SM, Steingrimsson O, Thormar H. In Vitro Inactivation of Chlamydia trachomatis by Fatty Acids and Monoglycerides. Antimicrobial Agents and Chemotherapy. 1998;42(9):2290-2294. doi:10.1128/AAC.42.9.2290

19. Skřrivanová E, Marounek M, Dlouhá G, Kaňka J. Susceptibility of Clostridium perfringens to C 2 -C 18 fatty acids. Letters in Applied Microbiology. 2005;41(1):77-81. doi:10.1111/j.1472-765X.2005.01709.x 
20. Shin S., Bajpai V., Kim H., Kang S. Antibacterial activity of eicosapentaenoic acid (EPA) against foodborne and food spoilage microorganisms. LWT - Food Science and Technology. 2007;40(9):1515-1519. doi:10.1016/j.1wt.2006.12.005

21. Greenway DL, Dyke KG, Greenway DL. Mechanism of the inhibitory action of linoleic acid on the growth of Staphylococcus aureus. Journal of general microbiology. 1979;115(1):233-245. doi:10.1099/00221287-115-1-233

22. Chamberlain NR, Mehrtens BG, Xiong Z, Kapral FA, Boardman JL, Rearick JI. Correlation of carotenoid production, decreased membrane fluidity, and resistance to oleic acid killing in Staphylococcus aureus 18Z. Infection and Immunity. 1991;59(12):4332-4437.

23. Carson DD, Daneo-Moore L. Effects of fatty acids on lysis of Streptococcus faecalis. The Journal of Bacteriology. 1980;141(3):1122-1126.

24. Thompson L, Cockayne A, Spiller RC. Inhibitory effect of polyunsaturated fatty acids on the growth of Helicobacter pylori: a possible explanation of the effect of diet on peptic ulceration. Gut. 1994;35(11):1557-15561. doi:10.1136/gut.35.11.1557

25. Dalia Seleem, Emily Chen, Bruna Benso, Vanessa Pardi, Ramiro M. Murata. In vitro evaluation of antifungal activity of monolaurin against Candida albicans biofilms. PeerJ. 2016;4(6):e2148. doi:10.7717/peerj.2148

26. Peterson ML, Schlievert PM. Glycerol monolaurate inhibits the effects of Gram-positive select agents on eukaryotic cells. Biochemistry. 2006;45(7):2387-2397.

doi:10.1021/bi051992u

27. Xiaowei LI, Manman G, Yizhao P, Liesu M, Shemin L. Molecular immune pathogenesis and diagnosis of COVID-19. Journal of Pharmaceutical Analysis. 2020 Mar 5. https://www.sciencedirect.com/science/article/pii/S2095177920302045\#! (accessed April $6,2020)$

28. Herold T, Jurinovic V, Arnreich C, Hellmuth JC, Von Bergwelt-Baildon M, Klein M, Weinberger T. Level of IL-6 predicts respiratory failure in hospitalized symptomatic COVID-19 patients. medRxiv doi: 10.1101/2020.04.01.20047381

29. Chen X, Zhao B, Qu Y, Chen Y, Xiong J, Feng Y, Men D, Huang Q, Liu Y, Yang B, Ding J, Li F. Detectable serum SARS-CoV-2 viral load (RNAaemia) is closely associated with drastically elevated interleukin 6 (IL-6) level in critically ill COVID-19 patients. medRxiv doi: 10.1101/2020.02.29.20029520 
30. Liu T, Zhang J, Yang Y, Ma H, Li Z, Zhang J, Cheng J, Zhang X, Zhao Y, Xia Z, Zhang $\mathrm{L}, \mathrm{Wu} \mathrm{G}, \mathrm{Yi}$ J. The potential role of IL-6 in monitoring severe cases of coronavirus disease 2019. medRxiv doi: 10.1101/2020.03.01.20029769

31. Sarkar NH, Charney J, Dion AS, Moore DH, Sarkar NH. Effect of human milk on the mouse mammary tumor virus. Cancer research. 1973;33(3):626-629. http://search.proquest.com/docview/81853637/.

32. Neyts J, Kristmundsdottir T, De Clercq E, Thormar H, Neyts J. Hydrogels Containing Monocaprin Prevent Intravaginal and Intracutaneous Infections With HSV-2 in Mice: Impact on the Search for Vaginal Microbicides. Journal of Medical Virology. 2000;61(1):107-110. doi:10.1002/(SICI)1096-9071(200005)61:1<107::AID-JMV17>3.0.CO;2-W

33. P. R. Cullis, M. J. Hope. Effects of fusogenic agent on membrane structure of erythrocyte ghosts and the mechanism of membrane fusion. Nature. 1978;271(5646):672-674. doi: $10.1038 / 271672 \mathrm{a} 0$

34. Witcher KJ, Novick RP, Schlievert PM, Witcher KJ. Modulation of immune cell proliferation by glycerol monolaurate. Clinical and diagnostic laboratory immunology. 1996;3(1):10-13. http://search.proquest.com/docview/78276504/.

35. Zhang MS, Sandouk A, Houtman JC. Glycerol Monolaurate (GML) Inhibits Human T Cell Signaling, Metabolism, and Function By Disrupting Lipid Dynamics. The Journal of Allergy and Clinical Immunology. 2017;139(2):AB269-AB269. doi:10.1016/j.jaci.2016.12.866

36. Qingsheng Li, Jacob D. Estes, Patrick M. Schlievert, et al. Glycerol monolaurate prevents mucosal SIV transmission. Nature. 2009;458(7241):1034-1038. doi:10.1038/nature07831

37. Baglivo M, Baronio M, Natalini G, Beccari T, Chiurazzi P, Fulcheri E, Petralia PP, Michelini S, Fiorentini G, Miggiano GA, Morresi A, Tonini G, Bertelli M. "Natural small molecules as inhibitors of coronavirus lipid-dependent attachment to host cells: a possible strategy for reducing SARS-COV-2 infectivity?" Acta Biomed. 2020 Mar 19;91(1):161-164. doi: 10.23750/abm.v91i1.9402 